\title{
Field-induced diastereomers for chiral separation
}

\author{
Andrey Yachmenev, ${ }^{1,2}$, * Jolijn Onvlee, $^{1}$ Emil Zak, ${ }^{1}$ Alec Owens, ${ }^{1,2,3}$ and Jochen Küpper ${ }^{1,2,4,1}$ \\ ${ }^{1}$ Center for Free-Electron Laser Science, Deutsches Elektronen-Synchrotron DESY, Notkestraße 85, 22607 Hamburg, Germany \\ ${ }^{2}$ Center for Ultrafast Imaging, Universität Hamburg, Luruper Chaussee 149, 22761 Hamburg, Germany \\ ${ }^{3}$ Department of Physics and Astronomy, University College London, Gower Street, WC1E 6BT London, United Kingdom \\ ${ }^{4}$ Department of Physics, Universität Hamburg, Luruper Chaussee 149, 22761 Hamburg, Germany
}

(Dated: October 28, 2019)

\begin{abstract}
A novel approach for the state-specific enantiomeric enrichment and the spatial separation of enantiomers is presented. Our scheme utilizes techniques from strong-field laser physics, specifically an optical centrifuge in conjunction with a static electric field, to create a chiral field with defined handedness. Molecular enantiomers experience unique rotational excitation dynamics and this can be exploited to spatially separate the enantiomers using electrostatic deflection. Notably, the rotational-state-specific enantiomeric enhancement and its handedness is fully controllable. To explain these effects, the conceptual framework of field-induced diastereomers of a chiral molecule is introduced and computationally demonstrated through robust quantum-mechanical simulations on the prototypical chiral molecule propylene oxide $\left(\mathrm{C}_{3} \mathrm{H}_{6} \mathrm{O}\right)$, for which ensembles with an enantiomeric excess of up to $30 \%$ were obtained.
\end{abstract}

Chirality is central to many chemical and biological processes. Its significance is emphasized by the fact that life on Earth is based on chiral biomolecules, which are all naturally selected with a single handedness that determines their functionality. Chiral molecules occur in structural forms known as enantiomers, which are mirror images of one another and, therefore, non-superimposable by translation and rotation. Given that molecular enantiomers have identical physical properties, neglecting the so-far unobserved effects of parity-violating weak interactions, but often strikingly different chemical behaviour, methods to distinguish and/or separate enantiomers are extremely important, particularly in areas such as drug design and pharmacology. Sources of cold chiral molecules in distinct enantiomeric states could improve measurements of electroweak interactions [1,3] and studies of collisional dynamics with chiral molecules [4].

Recently, a number of robust techniques for separating and purifying enantiomers in the gas phase from mixed chiral samples have been proposed [5] 9]. These developments are a consequence of the increased precision and control offered by gas-phase chemistry, which is providing exciting advances in the analysis and manipulation of chiral molecules. Sophisticated methods are now available for establishing the absolute configuration and enantiomeric excess $(e e)$, for example, employing phase-sensitive microwave spectroscopy [10, 11], photoelectron circular dichroism [12], Coulomb explosion imaging [13, 14], high-harmonic generation [15], or attosecond-time-resolved photoionization [16].

Here, we present a novel approach for coherent enantiomer-specific enrichment of rotational state populations of chiral molecules coupled to state-specific electricfield manipulation. Our scheme utilizes an optical centrifuge [17, 18, which is a linearly polarized light pulse that performs accelerated rotation about the direction of propagation, in conjunction with a static electric field

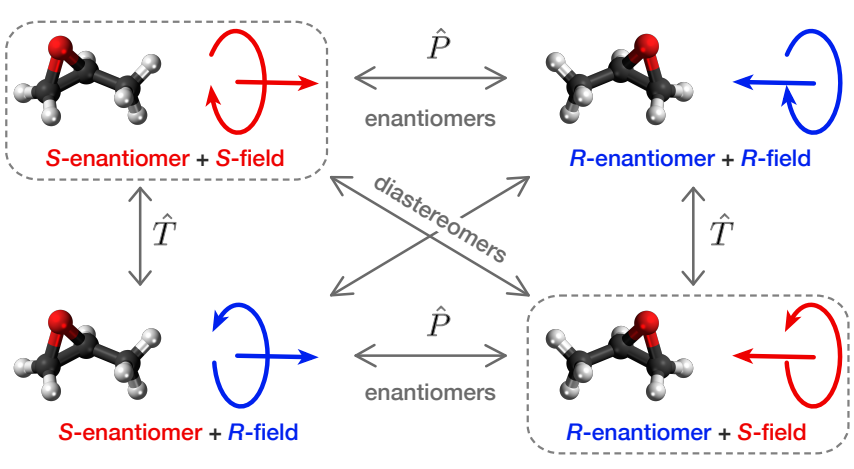

FIG. 1. Field-induced diastereomers of a chiral molecule interacting with a chiral electric field, i. e., a rotating optical field in conjunction with a static electric field along the rotation axis of the optical field.

along the light propagation direction. This yields a chiral electric field that induces unique rotational excitation dynamics in the different enantiomers . The rotationalstate-specific enantiomeric enhancement for either the left- or right-handed enantiomer is fully controllable by changing the duration of the optical centrifuge pulse, and it is subsequently transformed into spatial enhancement using the electrostatic deflector [19].

Similar to classical methods of chiral resolution, where a pure enantiomer of another chiral agent is introduced to form diastereomeric complexes with distinct physiochemical properties, we can create field-induced diastereomers of a chiral molecule, depicted in Fig. 1, by placing its enantiomers in a chiral electric field with defined handedness. The symmetry properties of the field-induced diastereomers are such that both, the molecule and the field enantiomers, are interconverted by the parity inversion operator $\hat{P}$, but time inversion $\hat{T}$ interchanges only the field enantiomers. Hence, two chiral centers emerge in the presence of the electric field with defined handedness, 
shown in the dashed panels in Fig. 1 and as for classical chemically-bound diastereomers, with distinct energies, the rotational dynamics of the enantiomers will differ.

The interaction of the enantiomers with a chiral electric field is described by the following time-dependent potential,

$$
V(t)=\frac{1}{4} \epsilon(t)^{2} \cos ^{2}(\omega t) V^{\mathrm{oc}}+\frac{1}{\sqrt{2}} \epsilon_{\mathrm{dc}} V^{\mathrm{dc}},
$$

with the optical-centrifuge contribution

$$
\begin{aligned}
V^{\mathrm{oc}} & =-2 \alpha D_{0,0}^{(0) *} \\
& -\Delta \alpha\left(e^{2 i \beta t} D_{-2,0}^{(2) *}+e^{-2 i \beta t} D_{2,0}^{(2) *}-\sqrt{\frac{2}{3}} D_{0,0}^{(2) *}\right) \\
& +\alpha_{x z}\left(e^{2 i \beta t} \mathcal{A}_{-2,1}^{(2)}+e^{-2 i \beta t} \mathcal{A}_{2,1}^{(2)}-\sqrt{\frac{2}{3}} \mathcal{A}_{0,1}^{(2)}\right) \\
& +i \alpha_{y z}\left(e^{2 i \beta t} \mathcal{S}_{-2,1}^{(2)}+e^{-2 i \beta t} \mathcal{S}_{2,1}^{(2)}-\sqrt{\frac{2}{3}} \mathcal{S}_{0,1}^{(2)}\right)
\end{aligned}
$$

and the dc-field component

$$
V^{\mathrm{dc}}=\mu_{x} \mathcal{A}_{0,1}^{(1)}+i \mu_{y} \mathcal{S}_{0,1}^{(1)}-\sqrt{2} \mu_{z} D_{0,0}^{(1) *} .
$$

Here, $\epsilon(t) \cos (\omega t)$ describes the linearly polarized carrier ac field of the optical centrifuge with the pulse envelope function $\epsilon(t)$ and angular frequency $\omega$ and the acceleration of the centrifuge circular rotation $\beta . \epsilon_{\mathrm{dc}}$ is the dc field strength. The electric field tensors $\mu_{\gamma}(\gamma=x, y, z), \alpha=$ $\left(\alpha_{x x}+\alpha_{y y}+\alpha_{z z}\right) / 3$ and $\Delta \alpha=\left(2 \alpha_{z z}-\alpha_{x x}-\alpha_{y y}\right) / \sqrt{6}$ are the permanent dipole moment, average static polarizability, and the static polarizability anisotropy, respectively, in the principal axis of inertia molecular frame. The symbol $D_{m, k}^{(J) *}$ denotes the complex-conjugated Wigner $D$-matrix, $\mathcal{A}_{m, k}^{(J)}=D_{m, k}^{(J) *}-D_{m,-k}^{(J) *}$, and $\mathcal{S}_{m, k}^{(J)}=D_{m, k}^{(J) *}+D_{m,-k}^{(J) *}$. The quantum numbers $k$ and $m$ correspond to the projection, in units of $\hbar$, of the total angular momentum $J$ onto the molecule-fixed $z$ axis and laboratory-fixed $Z$ axis, respectively. The full expression for the interaction potential is listed in the supplementary material.

The handedness of the chiral field in $(1)$ is defined by the relative signs of the dc field $\epsilon_{\mathrm{dc}}$ and the $\beta$ exponent, i. e., the direction of rotation of the optical centrifuge. Replacing one enantiomer with another changes the $V^{\text {oc }}$ and $V^{\mathrm{dc}}$ contributions in (2) and $(3)$ as this changes the sign of one component of the permanent molecular dipole moment as well as two components of the molecular polarizability tensor [20, 21].

This enantiomer-specific effect can be explained using a minimal model of a molecule as a three-level system of rotational states coupled by the dc and optical centrifuge fields. An example of two such three-level systems is shown in Fig. 2 for Raman transitions from the ground state $\left|J_{k \tau}, m\right\rangle=\left|0_{00}, 0\right\rangle$ to the excited states $\left|2_{00},-2\right\rangle$
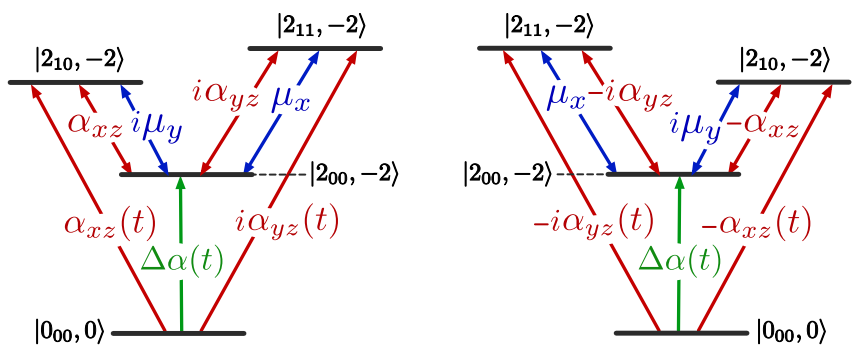

FIG. 2. Minimal model of the enantiomer-differentiating transitions in a chiral molecule. A system of three-rotational states originating from the ground state is coupled to the static electric field and optical centrifuge, for instance, by the molecular electric dipole moment $\mu_{y}$ and the polarizability parameters $\Delta \alpha$ and $\alpha_{x z}$ or, alternatively, by $\mu_{x}, \Delta \alpha$, and $\alpha_{y z}$, see (1)-(3). Here, $\Delta \alpha(t)=\Delta \alpha e^{2 i \beta t^{2}}$ and $\alpha_{a b}(t)=\alpha_{a b} e^{2 i \beta t^{2}}, a, b=x, y, z$.

and $\left|2_{10},-2\right\rangle$, and from the ground to the $\left|2_{00},-2\right\rangle$ and $\left|2_{11},-2\right\rangle$ states. Note that $\tau=0,1$ indicate the parity of the rotational wave function defined as $(-1)^{\tau}$. Assuming that the different enantiomers are related by inversion along the molecular $z$ axis leads to a sign change in the molecular dipole moment $\mu_{z}$ and the polarizabilities $\alpha_{x z}$ and $\alpha_{y z}$ between the different enantiomers. For simplicity, different interaction terms in (2) and (3) are abbreviated in Fig. 2 with the corresponding molecular polarizability $\Delta \alpha, \alpha_{x z}, \alpha_{y z}$ and dipole moment $\mu_{x}, \mu_{y}$ symbols.

We consider one transition band formed by the ground state $\left|0_{00}, 0\right\rangle$ and the two excited states $\left|2_{00},-2\right\rangle$ and $\left|2_{10},-2\right\rangle$ that, for simplicity, are assumed to have equal energy $E$ relative to the ground state. When the angular acceleration of the centrifuge is relatively slow, population transfer into both excited states, shown in Fig. 2 with the green and red colored upward arrows, will overlap in time: The optical centrifuge creates a superposition of states $\left|\psi_{0}\right\rangle=\cos (\chi t)\left|0_{00}, 0\right\rangle+$ $\Delta \alpha t e^{i \phi t}\left|2_{00},-2\right\rangle+\alpha_{x z} t e^{i \phi t}\left|2_{10},-2\right\rangle$, with the phase angle $\chi=\epsilon_{0}^{2} \sqrt{\Delta \alpha^{2}+\alpha_{x z}^{2}}$ and $\phi=0.5\left(\alpha \epsilon_{0}^{2}-E\right)$. The sign change of $\alpha_{x z}$ between enantiomers only changes the phase of $\left|\psi_{0}\right\rangle$ by $\pi$, but does not alter any transition probabilities. Note that we have also omitted the state superposition coefficients, which depend on the field parameters. This behaviour of the enantiomers in an optical field with twisted polarization has been described and investigated previously [20 22], mainly for determining the enantiomeric excess in mixtures.

The two excited states $\left|2_{00},-2\right\rangle$ and $\left|2_{10},-2\right\rangle$ are coupled to each other by virtue of the induced dipole interaction with the rapidly oscillating field of the optical centrifuge, $\alpha_{x z} \epsilon_{0}^{2} \equiv \mu_{z}^{\text {ind }} \epsilon_{0}$, and the permanent dipole interaction with the dc field, $i \mu_{y} \epsilon_{\mathrm{dc}}$. This quasi-static interaction, depicted with the red and blue colored doubleheaded arrows in Fig. 2, creates the superposition of states $\left|\psi_{0}\right\rangle=\cos (\chi t)\left|0_{00}, 0\right\rangle+0.5 \alpha_{x z} t^{2} c e^{i \theta}\left|2_{00},-2\right\rangle+$ 
$0.5 \Delta \alpha t^{2} c e^{-i \theta}\left|2_{10},-2\right\rangle$, with the coefficient $c \sim \mid \mu_{z}^{\text {ind }} \epsilon_{0}+$ $i \mu_{y} \epsilon_{\mathrm{dc}} \mid$ and the phase angle $\theta=\operatorname{atan} 2\left(\mu_{y} \epsilon_{\mathrm{dc}} / \mu_{z}^{\text {ind }} \epsilon_{0}\right)$. Note that this expression was obtained by truncating the exponential time-evolution operator at the second-order expansion term and is only valid for short timescales.

The probability for the molecule to be, for example, in the $|2\rangle=\left|2_{00},-2\right\rangle$ state is $P_{2} \propto$ $\left|\Delta \alpha e^{i \phi t}+0.5 \alpha_{x z} t^{2} c e^{i \theta_{R / S}}\right|^{2}$ with the sign of $\alpha_{x z}$ depending on the enantiomer and the phase angles $\theta_{R}$ and $\theta_{S}$ for the $R$ and $S$-enantiomers, respectively, which are related via $\theta_{R}=-\theta_{S}+\operatorname{sign}\left(\mu_{y} \epsilon_{\mathrm{dc}}\right) \pi$. The explicit expression for this probability can be derived as $P_{2} \propto \epsilon_{0}^{4} t^{2}\left(\Delta \alpha^{2}-\Delta \alpha \alpha_{x z} \mu_{y} \epsilon_{\mathrm{dc}} t\right)+\mathcal{O}\left(t^{4}\right)$. Similarly, the probability of being in the $|3\rangle=\left|2_{10},-2\right\rangle$ state is given by $P_{3} \propto \epsilon_{0}^{4} t^{2}\left(\alpha_{x z}^{2}+\Delta \alpha \alpha_{x z} \mu_{y} \epsilon_{\mathrm{dc}} t\right)+\mathcal{O}\left(t^{4}\right)$. Here, the product of the mutually orthogonal polarizability and permanent dipole moment $\alpha_{x z} \mu_{y}$ is independent of the choice of molecular axes, but changes sign between enantiomers, which gives rise to different populations of the $R$ and $S$-enantiomers in the $\left|2_{00},-2\right\rangle$ and $\left|2_{10},-2\right\rangle$ states.

Given the field configuration, i. e., the sign of the dc field $\pm \epsilon_{\mathrm{dc}}$ and the direction of centrifuge rotation $\pm \beta$, with $\pm \beta \equiv \pm t$ in the above equations, the sign of the total product $\alpha_{x z} \mu_{y} \epsilon_{\mathrm{dc}} t$ is determined by the absolute structure of the molecule in the chosen reference system. The molecular electric dipole moment and polarizability tensor can be obtained from an electronic structure calculation for a molecule of interest. Thus, given the field configuration it is possible to predict the handedness of the enantiomeric enhancement of the rotational state populations. The handedness can be reversed by inverting the direction of either the dc field or the centrifuge rotation, see Fig. 1. Indeed, for the $R$-enantiomer of propylene oxide in the principal axes system $\mu_{y}=0.69$, $\alpha_{x z}=-0.94$, and $\Delta \alpha=-6.26$, with all quantities in a.u., giving $P_{2}(R)<P_{2}(S)$ and $P_{3}(R)>P_{3}(S)$ for positive $\epsilon_{\mathrm{dc}}$ and $\beta$. This result is further confirmed by numerical simulations, vide infra.

From the expressions for $P_{2}$ and $P_{3}$, it is clear that a stronger dc field increases the enantiomeric enhancement. However, it also induces strong Stark repulsion in the excited states, which is neglected in our simple model, and hence decouple the two excited states in the superposition $\left|\psi_{0}\right\rangle$ produced by the optical centrifuge. A simple condition has been derived in the supplementary material to put an upper bound on the dc field for excited states of interest. In principle, the use of a stronger dc field for higher rotational excitations will improve the enantiomeric enhancement in the state populations, however, the difficulties associated with spatially separating high- $J$ states with inhomogeneous electric fields must be taken into consideration.

To quantitatively investigate the proposed scheme, fulldimensional quantum-mechanical simulations were performed for the prototypical chiral molecule propylene
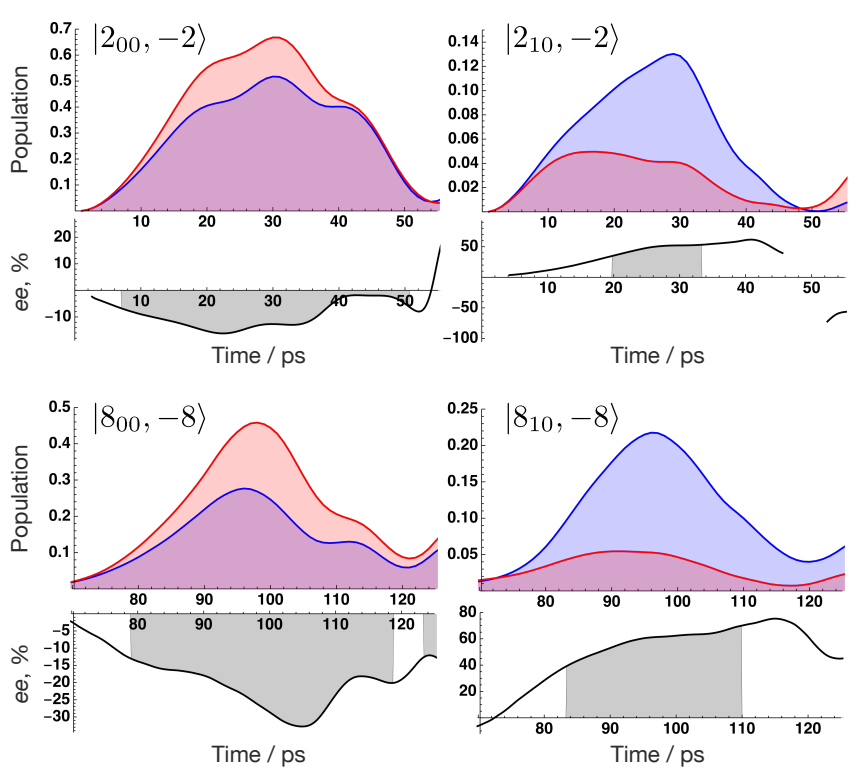

FIG. 3. Temporal evolution of the rotational state populations for the lowest-energy excited state pair for $J=2$ and $J=8$ for the $R$ (blue) and $S$ (red) enantiomers of propylene oxide, for a dc field of $10 \mathrm{kV} / \mathrm{cm}$. The enantiomeric excess ee of the rotational-state populations is shown beneath each population plot. $e e$ is plotted only for times when the population of either of the enantiomers in the final state is greater than $1 \%$ of the initial population in $\left|0_{00}, 0\right\rangle$ at $t=0$. The shaded gray regions indicate corresponding populations greater than $10 \%$.

oxide $\left(\mathrm{C}_{3} \mathrm{H}_{6} \mathrm{O}\right)$. The computational approach is based on highly accurate variational procedures capable of supporting high-resolution spectroscopy [23-25]; details are provided in the supplementary material. The optical centrifuge pulse was applied for a maximal duration of $140 \mathrm{ps}$ with the peak amplitude of the field $\epsilon_{0}=1 \times 10^{7} \mathrm{~V} / \mathrm{cm}$, chirp rate $\beta=(2 \pi c)^{2} \cdot 0.1 \mathrm{~cm}^{-2}$, and carrier frequency of the field $\omega=c /(2 \pi \cdot 800 \mathrm{~nm})$. The pulse envelope was modeled using a half Gaussian profile with a 140 ps halfwidth-at-half-maximum and a 1.5 ps smooth-cut applied at the beginning and the end of the truncated pulse. Several values for the dc field strength $\epsilon_{\mathrm{dc}}=1 \ldots 30 \mathrm{kV} / \mathrm{cm}$ were investigated and optimal results were obtained for $\epsilon_{\mathrm{dc}}=5 \ldots 10 \mathrm{kV} / \mathrm{cm}$. Finite-initial-temperature effects were modeled by averaging over the individual quantum wavepackets originating from different initial rotational states according to Boltzmann statistics. Calculations were performed for rotational temperatures of $T \leqslant 1 \mathrm{~K}$, with $T \lesssim 1 \mathrm{~K}$ directly achievable through supersonic expansion, whereas effective temperatures $T \leqslant 0.5 \mathrm{~K}$ are representative of state-selected molecular beams [19.

The dynamics of the enantiomers in a chiral field with $\epsilon_{\mathrm{dc}}=10 \mathrm{kV} / \mathrm{cm}$ are depicted by the time evolution of the rotational-state populations for the coupled pairs of the $\left|2_{00},-2\right\rangle$ and $\left|2_{10},-2\right\rangle$ states and the $\left|8_{00},-8\right\rangle$ and $\left|8_{10},-8\right\rangle$ states, see Fig. 3 The initial rotational temperature was set to $T_{i}=0 \mathrm{~K}$, i. e., all wavepacket population 

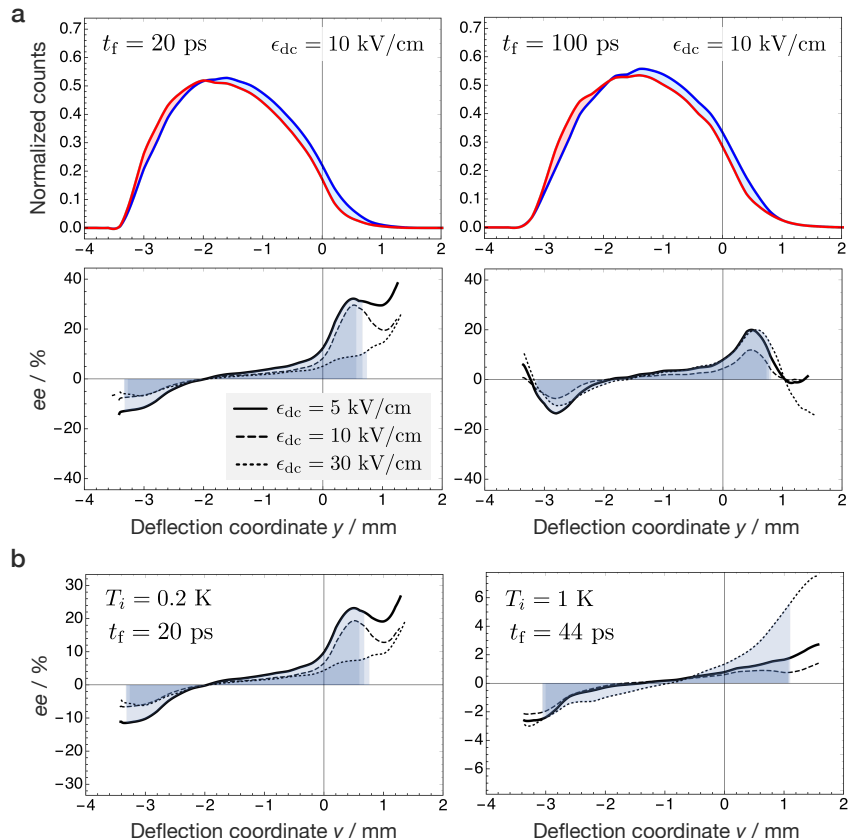

FIG. 4. (a) Deflection profiles and enantiomeric excess ee as a function of the vertical transverse beam position $y$, calculated for two release times from the optical centrifuge $t_{\mathrm{f}}=20 \mathrm{ps}$ and $100 \mathrm{ps}$, for the $R$ (blue) and $S$ (red) enantiomer of propylene oxide, for an initial rotational temperature of $T_{i}=0 \mathrm{~K} . y=0$ corresponds to the center of the undeflected molecular beam and the deflector's field strength increases in the negative $y$ direction, down to $y \approx-3 \mathrm{~mm}$ [26]. (b) Enantiomeric excess $e e$ as a function of the deflection coordinate $y$, computed at release times $t_{\mathrm{f}}=20 \mathrm{ps}$ and $44 \mathrm{ps}$ for finite initial rotational temperatures of $T_{i}=0.2 \mathrm{~K}$ and $1 \mathrm{~K}$, respectively. ee is plotted only for deflection positions $y$ at which the ratio between the column density for either of the enantiomers and the total column density at $y=0 \mathrm{~mm}$ is greater than $1 \%$. Filled areas show ratios greater than $10 \%$.

started in the rovibrational ground state $\left|0_{00}, 0\right\rangle$. The optical centrifuge excited the molecules via $\Delta J=2, \Delta m=-2$ rotational Raman transitions and a significant amount of the wavepacket population for the $R$-enantiomer was transferred into the $\left|2_{10},-2\right\rangle$ state at time $t \approx 30$ ps and into the $\left|8_{10},-8\right\rangle$ state at $t \approx 100 \mathrm{ps}$. This produced an excess of the $R$-enantiomer in the population of these states, while an excess of the $S$-enantiomer is evident in the population of the $\left|2_{00},-2\right\rangle$ and $\left|8_{00},-8\right\rangle$ states. To quantify the population difference, the time evolution of the enantiomeric excess ee $=(p(R)-p(S)) /(p(R)+p(S)) \cdot 100 \%$ of the respective rotational state populations $p(R)$ and $p(S)$ is shown in Fig. 3. As expected, the enantiomeric enhancement is more pronounced at higher rotational excitations, owing to the smaller energy gap between the $\left|8_{00},-8\right\rangle$ and $\left|8_{10},-8\right\rangle$ states. Furthermore, the absolute handedness of the enantiomeric excess in these states confirms the predictions of the simplified three-level model, vide supra.
The enantiomeric enhancement in the rotational state populations can be converted to spatial enrichment by releasing the molecules from the optical centrifuge at optimal times before entering the electrostatic deflector, where molecules are spatially dispersed depending on their rotational state. The rotational state populations of the $R$ and $S$-enantiomers were extracted from quantum-mechanical simulations for different durations $t_{\mathrm{f}}$ of the optical centrifuge pulse. Then, their deflection profiles were simulated [27, 28] for a typical experimental setup [26], details are provided in the supplementary material. The resulting vertical beam profiles are shown in Fig. 4 for beams with initial rotational temperatures of $T_{i}=0,0.2,1 \mathrm{~K}$.

In Fig. 4 a, deflection profiles are shown for two optimal release times $t_{\mathrm{f}}=20 \mathrm{ps}$ and $100 \mathrm{ps}$ for an initial $T_{i}=0 \mathrm{~K}$. Here, the difference in the profiles for the $R$ and $S$-enantiomers are largely caused by the enhanced population of the $\left|2_{00},-2\right\rangle$ or $\left|8_{00},-8\right\rangle$ state in the $S$-enantiomer, which both have stronger Stark shifts than the respective $\left|2_{10},-2\right\rangle$ and $\left|8_{10},-8\right\rangle$ states, which produced an excess of the $R$-enantiomer. Consequently, the $S$-enantiomer is more strongly deflected toward the larger dc field, i. e., in the negative $y$ direction. Since population of the $\left|2_{00},-2\right\rangle$ or $\left|8_{00},-8\right\rangle$ state in both enantiomers is larger than that of the $\left|22_{10},-2\right\rangle$ or $\left|8_{10},-8\right\rangle$ state (see Fig. 3), deflection enhances the concentration of $S$-enantiomers in the lower part of the deflected molecular beam, i. e., on the left-hand side of the plot.

The spatial enantiomeric excess for different values of the dc field is illustrated for $T_{i}=0 \mathrm{~K}$ below the deflection profiles in Fig. $4 \mathrm{a}$ and for $T_{i}=0.2$ and $1 \mathrm{~K}$ in Fig. $4 \mathrm{~b}$. An optimal dc field between $\epsilon_{\mathrm{dc}}=5$ and $10 \mathrm{kV} / \mathrm{cm}$ for $T_{i}=0$ and $0.2 \mathrm{~K}$ provides maximal enantiomeric enrichment of about $30 \%$. The profile for $T_{i}=0.2 \mathrm{~K}$ is similar to that for $T_{i}=0 \mathrm{~K}$ and is mostly dominated by the dynamics of the $\left|2_{00}, 0\right\rangle$ and $\left|2_{10}, 0\right\rangle$ states with the absolute molecular intensity scaled by the corresponding thermal Boltzmann factor. The enhancement diminishes for stronger dc fields of $\epsilon_{\mathrm{dc}}=30 \mathrm{kV} / \mathrm{cm}$ due to the decoupling of the $\left|2_{00}, 0\right\rangle$ and $\left|2_{10}, 0\right\rangle$ states caused by strong Stark repulsion. For higher initial temperatures, excitation with the optical centrifuge leads to a more spectrally broadened rotational wavepacket. For $T_{i}=1 \mathrm{~K}$ the wavepackets are composed of a large number of rotational states, with each state reaching its largest $e e$ in population at a different time $t_{\mathrm{f}}$. This significantly reduces the enantiomeric excess to $6 \%$. In addition, since many states with higher $k$ quanta are initially populated, they exhibit smaller repulsive shifts and, therefore, display an increased $e e$ at stronger dc fields. This is evidenced by the unexpected rise of enrichment at $\epsilon_{\mathrm{dc}}=30 \mathrm{kV} / \mathrm{cm}$ for $T_{i}=1 \mathrm{~K}$. The results of Fig. 4 calculated for different release times $0<t_{\mathrm{f}}<100 \mathrm{ps}$ are shown through animated videos for different initial temperatures $T_{i}$ in the supplementary material [29 38].

In the presented scheme for the spatial separation of enantiomers, the enantiomeric enhancement is associated 
with the distinct rotational excitation dynamics of the field-induced diastereomers of a chiral molecule placed in a chiral field of defined handedness. The chiral field is created by the combination of an optical centrifuge laser pulse with a dc field parallel or antiparallel to the light's propagation direction. The enhancement of rotational state populations can be exploited to spatially separate the $R$ and $S$ enantiomers using electrostatic deflection techniques. This has been confirmed through robust numerical simulations for propylene oxide. A simple threestate model was established to explain these effects in a similar manner to microwave three-wave mixing [5, 6, i.e., as interference of the three-photon pathways in molecular enantiomers. The enantiomer-differentiating effect, captured, e.g., by $\Delta \alpha-\alpha_{x z} \mu_{y} \epsilon_{\mathrm{dc}} t$ or $\alpha_{x z}-\Delta \alpha \mu_{y} \epsilon_{\mathrm{dc}} t$, originates in the coupling of the odd-parity molecular polarizability $\alpha_{x z}$ with the chiral field, which is both time- and parity-odd. In comparison to microwave threewave mixing, the presented approach can offer higher degrees of population transfer, possibly up to $80 \%$, see Fig. 3, which is achieved by the combination of ultracold deflected molecular beams and a focused high-intensity chirped laser field to drive Raman-type transitions in a three-level system.

Furthermore, our scheme can clearly distinguish between right-handed and left-handed enantiomers and, in principle, is applicable to any chiral molecule. The implementation generally requires optimization of the laser parameters and dc field strength [24, 39,41]. Given that only modest rotational excitation is sufficient, application of the optical centrifuge to larger molecules should not be overly problematic.

This work has been supported by the Deutsche Forschungsgemeinschaft (DFG) through the priority program "Quantum Dynamics in Tailored Intense Fields" (QUTIF, SPP1840, KU 1527/3, YA 610/1), through the Clusters of Excellence "Center for Ultrafast Imaging" (CUI, EXC 1074, ID 194651731) and "Advanced Imaging of Matter" (AIM, EXC 2056, ID 390715994), and by the European Research Council under the European Union's Seventh Framework Programme (FP7/2007-2013) through the Consolidator Grant COMOTION (ERC614507-Küpper). J.O. and A.O. gratefully acknowledge fellowships of the Alexander von Humboldt Foundation.

andrey.yachmenev@cfel.de jochen.kuepper@cfel.de molecule-imaging.org

https://www.controlled-

[1] C. Daussy, T. Marrel, A. Amy-Klein, C. T. Nguyen, C. J. Bordé, and $\mathrm{C}$. Chardonnet, Limit on the parity nonconserving energy difference between the enantiomers of a chiral molecule by laser spectroscopy, Phys. Rev. Lett. 83, 1554 (1999).

[2] M. Quack, J. Stohner, and M. Willeke, High-resolution spectroscopic studies and theory of parity violation in chiral molecules, Annu. Rev. Phys. Chem. 59, 741 (2008)

[3] M. Schnell and J. Küpper, Tailored molecular samples for precision spectroscopy experiments, Faraday Disc. 150, $33(2011)$

[4] A. Lombardi and F. Palazzetti, Chirality in molecular collision dynamics, J. Phys.: Condens. Matter 30, 063003 (2018).

[5] S. Eibenberger, J. Doyle, and D. Patterson, Enantiomerspecific state transfer of chiral molecules, Phys. Rev. Lett. 118, 123002 (2017)

[6] C. Pérez, A. L. Steber, S. R. Domingos, A. Krin, D. Schmitz, and M. Schnell, Coherent enantiomerselective population enrichment using tailored microwave fields, Angew. Chem. Int. Ed. 56, 12512 (2017)

[7] K. Banerjee-Ghosh, O. B. Dor, F. Tassinari, E. Capua, S. Yochelis, A. Capua, S.-H. Yang, S. S. P. Parkin, S. Sarkar, L. Kronik, L. T. Baczewski, R. Naaman, and Y. Paltiel, Separation of enantiomers by their enantiospecific interaction with achiral magnetic substrates, Science 360, 1331 (2018)

[8] S. B. Wang and C. T. Chan, Lateral optical force on chiral particles near a surface, Nat. Commun. 5, 3307 (2014).

[9] A. Hayat, J. P. B. Mueller, and F. Capasso, Lateral chirality-sorting optical forces, PNAS 112, 13190 (2015)

[10] D. Patterson, M. Schnell, and J. M. Doyle, Enantiomerspecific detection of chiral molecules via microwave spectroscopy, Nature 497, 475 (2013)

[11] S. R. Domingos, C. Pérez, and M. Schnell, Sensing chirality with rotational spectroscopy, Annu. Rev. Phys. Chem. 69, 499 (2018)

[12] M. H. M. Janssen and I. Powis, Detecting chirality in molecules by imaging photoelectron circular dichroism, Phys. Chem. Chem. Phys. 16, 856 (2014)

[13] M. Pitzer, M. Kunitski, A. S. Johnson, T. Jahnke, H. Sann, F. Sturm, L. P. H. Schmidt, H. Schmidt-Böcking, R. Dörner, J. Stohner, J. Kiedrowski, M. Reggelin, S. Marquardt, A. Schießer, R. Berger, and M. S. Schöffler, Direct determination of absolute molecular stereochemistry in gas phase by Coulomb explosion imaging, Science 341, 1096 (2013)

[14] P. Herwig, K. Zawatzky, M. Grieser, O. Heber, B. JordonThaden, C. Krantz, O. Novotný, R. Repnow, V. Schurig, D. Schwalm, Z. Vager, A. Wolf, O. Trapp, and H. Kreckel, Imaging the absolute configuration of a chiral epoxide in the gas phase, Science 342, 1084 (2013)

[15] R. Cireasa, A. E. Boguslavskiy, B. Pons, M. C. H. Wong, D. Descamps, S. Petit, H. Ruf, N. Thiré, A. Ferré, J. Suarez, J. Higuet, B. E. Schmidt, A. F. Alharbi, F. Légaré, V. Blanchet, B. Fabre, S. Patchkovskii, O. Smirnova, Y. Mairesse, and V. R. Bhardwaj, Probing molecular chirality on a sub-femtosecond timescale, Nat. Phys. 11, 654 (2015).

[16] S. Beaulieu, A. Comby, A. Clergerie, J. Caillat, D. Descamps, N. Dudovich, B. Fabre, R. Géneaux, F. Légaré, S. Petit, B. Pons, G. Porat, T. Ruchon, R. Taïeb, V. Blanchet, and Y. Mairesse, Attosecond-resolved photoionization of chiral molecules, Science 358, 1288 (2017)

[17] J. Karczmarek, J. Wright, P. Corkum, and M. Ivanov, Optical centrifuge for molecules, Phys. Rev. Lett. 82, 3420 (1999).

[18] D. M. Villeneuve, S. A. Aseyev, P. Dietrich, M. Spanner, M. Y. Ivanov, and P. B. Corkum, Forced molecular rotation in an optical centrifuge, Phys. Rev. Lett. 85, 542 
(2000)

[19] Y.-P. Chang, D. A. Horke, S. Trippel, and J. Küpper, Spatially-controlled complex molecules and their applications, Int. Rev. Phys. Chem. 34, 557 (2015), arXiv:1505.05632 [physics]

[20] A. Yachmenev and S. N. Yurchenko, Detecting chirality in molecules by linearly polarized laser fields, Phys. Rev. Lett. 117, 033001 (2016)

21] I. Tutunnikov, E. Gershnabel, S. Gold, and I. Sh. Averbukh, Selective orientation of chiral molecules by laser fields with twisted polarization, J. Phys. Chem. Lett. 9, 1105 (2018)

[22] A. A. Milner, J. A. M. Fordyce, I. MacPhail-Bartley, W. Wasserman, V. Milner, I. Tutunnikov, and I. S. Averbukh, Controlled enantioselective orientation of chiral molecules with an optical centrifuge, Phys. Rev. Lett. 122, 223201 (2019).

[23] A. Owens and A. Yachmenev, RichMol: A general variational approach for rovibrational molecular dynamics in external electric fields, J. Chem. Phys. 148, 124102 (2018), arXiv:1802.07603 [physics.chem-ph]

[24] A. Owens, A. Yachmenev, and J. Küpper, Coherent control of the rotation axis of molecular superrotors, J. Phys. Chem. Lett. 9, 4206 (2018), arXiv:1807.04016 [physics].

[25] A. Owens, A. Yachmenev, S. N. Yurchenko, and J. Küpper, Climbing the Rotational Ladder to Chirality, Phys. Rev. Lett. 121, 193201 (2018), arXiv:1802.07803 [physics]

[26] J. S. Kienitz, K. Długołęcki, S. Trippel, and J. Küpper, Improved spatial separation of neutral molecules, J. Chem. Phys. 147, 024304 (2017), arXiv:1704.08912 [physics]

[27] Y.-P. Chang, F. Filsinger, B. Sartakov, and J. Küpper, CMIsTARK: Python package for the stark-effect calculation and symmetry classification of linear, symmetric and asymmetric top wavefunctions in dc electric fields, Comp. Phys. Comm. 185, 339 (2014), arXiv:1308.4076 [physics]

[28] F. Filsinger, J. Küpper, G. Meijer, L. Holmegaard, J. H. Nielsen, I. Nevo, J. L. Hansen, and H. Stapelfeldt, Quantum-state selection, alignment, and orientation of large molecules using static electric and laser fields, J. Chem. Phys. 131, 064309 (2009), arXiv:0903.5413 [physics]

[29] See supplemental material for computational details, which includes additional references given in [30-38].

[30] T. H. Dunning, Gaussian basis sets for use in correlated molecular calculations. I. The atoms boron through neon and hydrogen, J. Chem. Phys. 90, 1007 (1989).

[31] R. A. Kendall, T. H. Dunning, Jr., and R. J. Harrison, Electron affinities of the first-row atoms revisited. Systematic basis sets and wave functions, J. Chem. Phys. 96,
6796 (1992)

[32] CFOUR, Coupled-Cluster techniques for Computational Chemistry, a quantum chemical program package written by J. F. Stanton, J. Gauss, M. E. Harding, and P. G. Szalay with contributions from A. A. Auer, R. J. Bartlett, U. Benedikt, C. Berger, D. E. Bernholdt, Y. J. Bomble, L. Cheng, O. Christiansen, M. Heckert, O. Heun, C. Huber, T.-C. Jagau, D. Jonsson, J. Jusélius, K. Klein, W. J. Lauderdale, D. A. Matthews, T. Metzroth, L. A. Mück, D. P. O'Neill, D. R. Price, E. Prochnow, C. Puzzarini, K. Ruud, F. Schiffmann, W. Schwalbach, S. Stopkowicz, A. Tajti, J. Vázquez, F. Wang, J. D. Watts, and the integral packages MOLECULE (J. Almlöf and P. R. Taylor), PROPS (P. R. Taylor), ABACUS (T. Helgaker, H. J. Aa. Jensen, P. Jørgensen, and J. Olsen), and ECP routines by A. V. Mitin and C. van Wüllen. For the current version, see http://www.cfour.de (2018).

[33] J. M. Hollas, High Resolution Spectroscopy, 2nd ed. (John Wiley \& Sons, Chichester, 1982).

[34] J. D. Swalen and D. R. Herschbach, Internal barrier of propylene oxide from the microwave spectrum. I, J. Chem. Phys. 27, 100 (1957)

[35] R. A. Creswell and R. Schwendeman, Centrifugal distortion constants and structural parameters of methyl oxirane, J. Mol. Spectrosc. 64, 295 (1977)

[36] B. A. McGuire, P. B. Carroll, R. A. Loomis, I. A. Finneran, P. R. Jewell, A. J. Remijan, and G. A. Blake, Discovery of the interstellar chiral molecule propylene oxide $\left(\mathrm{CH}_{3} \mathrm{CHCH}_{2} \mathrm{O}\right)$, Science 352, 1449 (2016).

[37] S. Fleischer, Y. Khodorkovsky, Y. Prior, and I. S. Averbukh, Controlling the sense of molecular rotation, New J. Phys. 11, 105039 (2009)

|38 K. Kitano, H. Hasegawa, and Y. Ohshima, Ultrafast angular momentum orientation by linearly polarized laser fields, Phys. Rev. Lett. 103, 223002 (2009)

[39] S. Trippel, T. Mullins, N. L. M. Müller, J. S. Kienitz, K. Długołęcki, and J. Küpper, Strongly aligned and oriented molecular samples at a $\mathrm{kHz}$ repetition rate, $\mathrm{Mol}$. Phys. 111, 1738 (2013), arXiv:1301.1826 [physics]

[40] A. Chatterley, E. T. Karamatskos, C. Schouder, L. Christiansen, A. V. Jörgensen, T. Mullins, J. Küpper, and H. Stapelfeldt, Switched wave packets with spectrally truncated chirped pulses, J. Chem. Phys. 148, 221105 (2018), arXiv:1803.03953 [physics.chem-ph]

[41] S. Kerbstadt, K. Eickhoff, T. Bayer, and M. Wollenhaupt, Odd electron wave packets from cycloidal ultrashort laser fields, Nat. Commun. 10, 658 (2019). 